\title{
Binocular Image Sequence Analysis: Integration of Stereo Disparity and Optic Flow for Improved Obstacle Detection and Tracking
}

\author{
Yingping Huang ${ }^{1}$ and Ken Young ${ }^{2}$ \\ ${ }^{1}$ International Automotive Research Centre (IARC), Warwick Manufacturing Group, University of Warwick, Coventry CV4 7AL, UK \\ ${ }^{2}$ International Manufacturing Research Centre, Warwick Manufacturing Group, University of Warwick, Coventry CV4 7AL, UK
}

Correspondence should be addressed to Yingping Huang, huang_y@wmgmail.wmg.warwick.ac.uk

Received 28 August 2007; Revised 15 February 2008; Accepted 28 March 2008

Recommended by Ati Baskurt

Binocular vision systems have been widely used for detecting obstacles in advanced driver assistant systems (ADASs). These systems normally utilise disparity information extracted from left and right image pairs, but ignore the optic flows able to be extracted from the two image sequences. In fact, integration of these two methods may generate some distinct benefits. This paper proposes two algorithms for integrating stereovision and motion analysis for improving object detection and tracking. The basic idea is to fully make use of information extracted from stereo image sequence pairs captured from a stereovision rig. The first algorithm is to impose the optic flows as extra constraints for stereo matching. The second algorithm is to use a Kalman filter as a mixer to combine the distance measurement and the motion displacement measurement for object tracking. The experimental results demonstrate that the proposed methods are effective for improving the quality of stereo matching and three-dimensional object tracking.

Copyright (c) 2008 Y. Huang and K. Young. This is an open access article distributed under the Creative Commons Attribution License, which permits unrestricted use, distribution, and reproduction in any medium, provided the original work is properly cited.

\section{INTRODUCTION}

Driving safety is a serious issue for our society. Statistical data shows about 40 million road accidents happen every year in Europe, and 1.5 million peoples are killed by these accidents. Analysis of these accidents indicates that about 70 percent of serious injury accidents are caused by lack of driver attention. This fact necessitates developing advanced driver assistance systems (ADAS) for modern vehicles. The target of ADAS is to make the vehicle aware of its surroundings and allow it to take proper action in response to dangerous situations.

Vision-based sensing systems have been widely adapted for object detection in modern intelligent vehicles. Stereovision and motion analysis are two common computer vision techniques for extracting objects from sets of images. In stereovision, two images captured at the same time but from different positions are used to compute depth information of objects [1-4]. The motion analysis method segments objects according to their optic flows (motion vectors) by analysing two or more consecutive images taken with the same camera
[5-8]. These two methods have been researched separately, but very little attention has been paid to integrating them. In fact, integration of these two methods may generate some distinct benefits. This paper proposes two fusion algorithms for improving object detection and tracking. The basic idea is to fully make use of two pairs of image sequences captured from a stereovision rig, that is disparity from left and right pair images and optic flows from consecutive images.

For a stereovision-based object detection system, a key task is locating the image of a scene point in the left and right image pair, that is correspondence matching. This process generates a disparity map which has a crucial effect on the detection performance. In our previous stereovision work $[1,2]$, the correspondence matching is achieved in terms of the greyscale similarity of two image blocks centred at the points to be matched. The greyscale similarity of two window blocks is assessed by the sum of absolute differences (SAD) [2] or by the normalised cross correlation coefficient [1] between them. However, the correspondence matching obtained by this method is not enough to guarantee a true 
correspondence matching and may generate a number of false matching points because there are often many points in an image that have the same or very similar intensities. In this paper, we propose a method to improve the quality of correspondence matching. In this method, optic flows obtained from the left and right image sequences are used as extra constraints for correspondence matching.

In addition, object tracking is important to achieve more accurate object speeds in both longitudinal and lateral directions. Use of a Kalman filter is an effective method for object tracking and requires measurements to update prediction results. It is known that stereovision is able to measure object longitudinal distance while motion analysis is capable of measuring the lateral displacements of an object. This implies that a Kalman filter provides a natural way to fuse stereovision and motion analysis by using them as measurement tools. In this paper, we will also examine how stereovision and motion analysis can be fused by a Kalman filter for object tracking.

Stereomotion integration has been studied in a theoretical manner for extracting the relationship between them. Waxman and Duncan [9] claimed that "stereovision and motion analyses can be combined in such a way that each can help "to overcome the other's shortcomings" and proposed a 5 -step fusion strategy for extracting shape information. One important result was a correlation between relative image flow (i.e., binocular difference flow) and stereo disparity. Their implementations were limited and based on scenes consisting of white objects covered by black dots. Li et al. $[10,11]$ presented a method for recovering $3 \mathrm{D}$ translational motion and structure from binocular image flows. Translation motion parameters were first determined from a group of linear equations generated from measured optical flows of a number of image points. The estimated motion parameters were then used to find the correspondence between binocular image pairs. Results were presented upon laboratory scenes consisting of surfaces covered with either grids or regular patterns of black dots. Zhang and Kambhamettu [12] have proposed twoalgorithms for computing dense three-dimensional scene flow and structure from multiview image sequences. Stereo constraints under a multiview camera setup were investigated and utilized in model-based algorithms and extended gradient-based algorithms. Sudhir et al. [13] and Clifford and Nasrabadi [14] used Markov random fields to combine the stereo and motion. In Sudhir's method, the discontinuities are used to predict and enforce the preservation of stereo correspondences through the motion estimates. They presented the computed optical flow and stereo disparity on some tailored images. These researches did not consider object grouping issues, and therefore are difficult to apply in real scenarios. Tirumalai et al. [15] presented an approach for incremental refinement of disparity maps for a mobile robot. They utilized the motion vectors calculated from a least median of squares (LMS)based algorithm for recursive disparity prediction and refinement. More recently, Dang et al. [16] proposed using a Kalman filter for fusing optical flow and stereo disparity for object tracking. In their method, a complicated extended Kalman filter is used for the iteration process. In our study, we propose a decoupling filtering method by decomposing the state vector into three groups. The decomposed Kalman filter for each group of vectors has a linear relation, leading to a greatly simplified computation. In addition, the Kalman filter proposed by Dang et al. used a simplified motion prediction equation by considering accelerations as noise. This approach can reduce the computational complexity of the extended Kalman filter, but will bring a bigger error to the tracking results when the car is moving with acceleration. In our work, we use a complete motion equation as the prediction equation. The experimental results demonstrate that our Kalman filter is able to effectively track the distance and speed for all dynamic cases.

This paper consists of four sections. Section 2, following this introduction, describes fusion algorithm I that is fusing optic flows to stereo matching for improving the quality of the disparity map. Section 3 presents fusion algorithm II that introduces how stereovision and motion analysis can be fused by a Kalman filter for object tracking. Section 4 gives the conclusions.

\section{INTEGRATING OPTIC FLOWS WITH STEREO MATCHING}

For a stereovision-based object detection system, two images (left and right) sequences can be simultaneously acquired. This enables acquisition of a pair of motion fields by analyzing the left and right image sequences. Since true motion fields are not accessible, optical flow representing the displacement of corresponding points in consecutive images is used as an approximation. Optic flows for the same target calculated from the left and right image sequences should be similar. This similarity of optic flows can be taken as extra matching constraints. To fulfill this, we firstly need to calculate the optic flows for the left and right image sequences.

\subsection{Optic flows of moving obstacles}

Many approaches have been designed for obtaining optic flows for moving objects from image sequences [17]. Basically, these approaches can be split into two categories, that is, spatiotemporal gradient-based techniques and correspondence matching techniques. The spatiotemporal gradientbased techniques calculate optic flows based on assumptions such as globe smoothness or direction smoothness, and theoretical mathematical derivation. These assumptions cannot be guaranteed to be true in practical situations. The correspondence matching-based techniques detect optic flows by searching for correspondence points between consecutive images. These are, therefore, more suitable for dynamic road scene images. In this research, we employ a correspondence matching approach based on greyscale similarity to acquire optic flows.

In our previous work [2], we have designed an edge indexed stereo matching method. A summary for this method is as follows.

(i) Only conduct correspondence matching for edge points. 

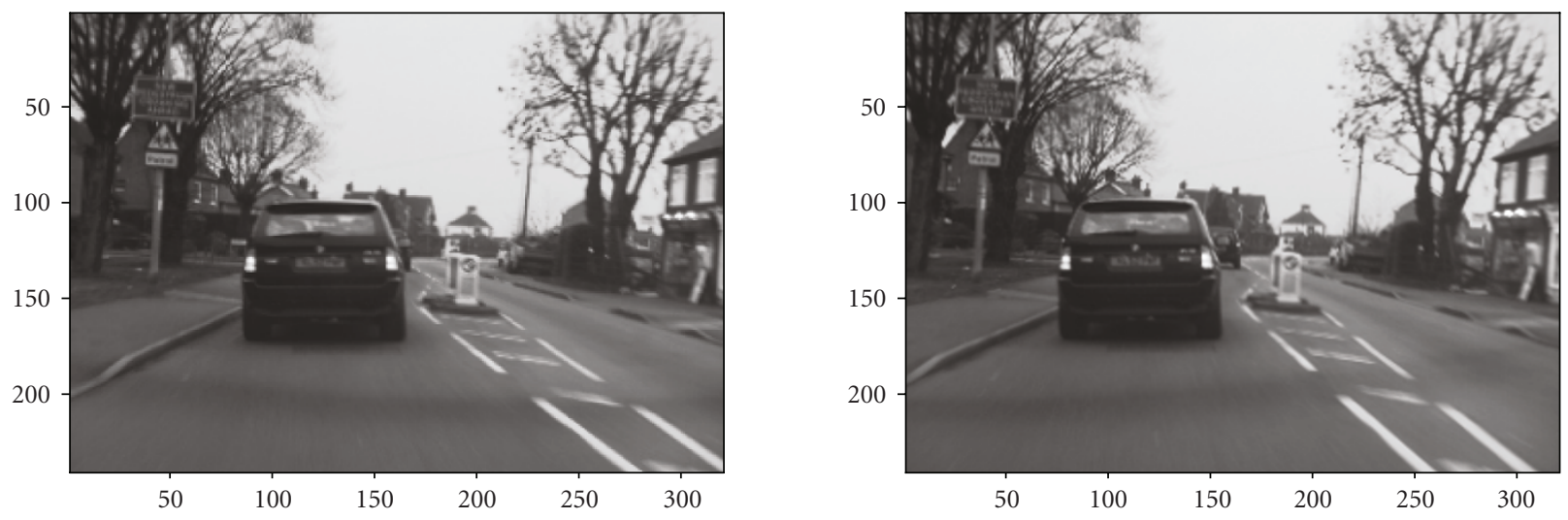

(a) The stereo image pair at frame 60
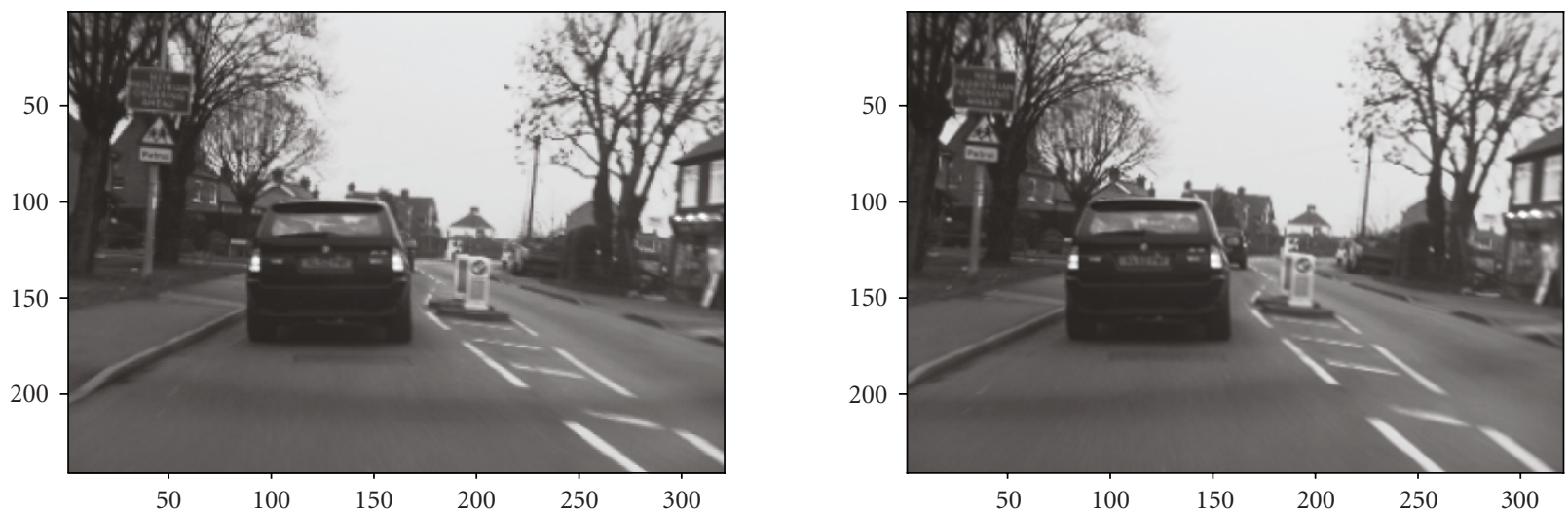

(b) The stereo image pair at frame 61

Figure 1: Consecutive stereo image sequence pairs.

(ii) Normalized cross correlation coefficients are used as a measure of greyscale similarity of two window blocks centred at the points to be assessed. The correspondence points are considered as those with the maximum cross correlation coefficients, which must be greater than a predefined threshold.

(iii) A quadratic interpolation was introduced to achieve a subpixel disparity estimation. This is to improve the depth resolution so that a higher depth detection accuracy can be achieved.

In this research, we also use the same method to calculate optic flows for the left and right image sequences. Some alterations have been made as follows.

(i) The correspondence searching is conducted in consecutive images rather than left and right image pairs.

(ii) No epipolar constraint can be applied, therefore the range of correspondence searching is within a square area centred at the seed point position.

(iii) For the right image sequence, the optic flows will be calculated on all candidate points to be matched with the seed points in the left image.
Figure 1 shows two stereo image pairs captured at consecutive time frames. A Canny edge detector has been used to achieve edge information as detailed as possible. Figure 2 shows the Canny detection results of the stereo image pair at frame 60. The edge points in these two edge images are used as seed points to search the correspondence points in the second stereo image pair. That is, the stereo image pair at frame 60 is used as the reference images to determine the optic flows. The threshold of the Canny filter has an influence on the amount of points to be matched. In this research, only edge points have been selected to conduct the stereo matching in order to reduce the computational burden. Actually, conducting matching on all points is ideal for object segmentations because it gives a dense disparity map. Calculated optic flows are shown in Figure 3 where the optic flows for each edge point are decomposed into displacements in horizontal $(X)$ and vertical $(Y)$ directions. Figures 3(a) and 3(b) show the horizontal displacements for the left and right images while Figures 3(c) and 3(d) show vertical displacements. A colour scheme is used to visualise the displacement vector fields. The colour bars indicate the quantitative relationship between the displacement and the colour. The maximum displacement, that is matching 
Left image edge

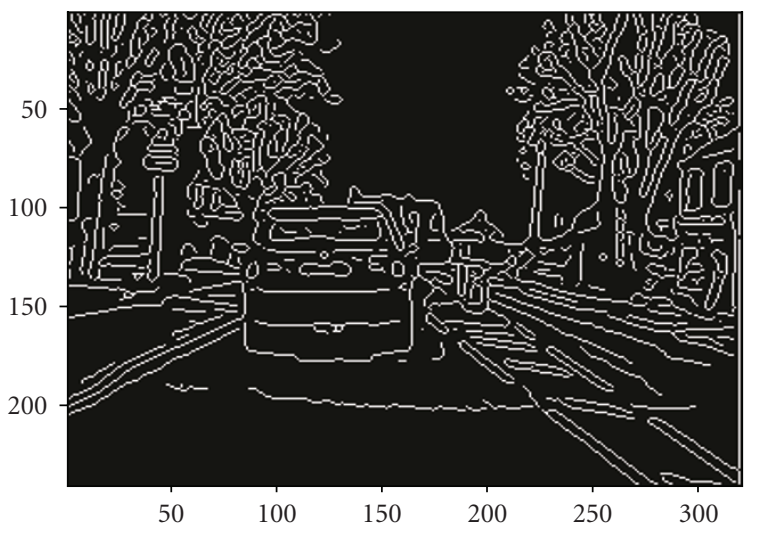

(a)

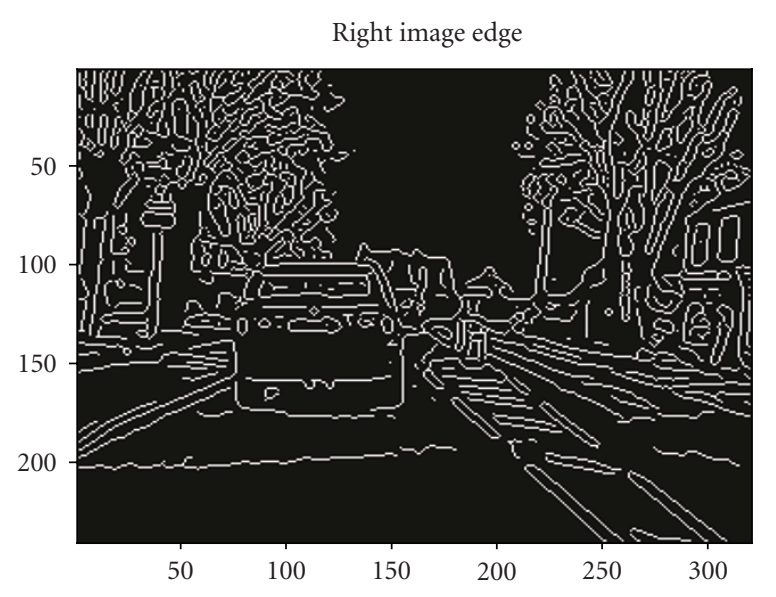

(b)

FIGURE 2: Edge image of the stereo image pair in Figure 1(a).

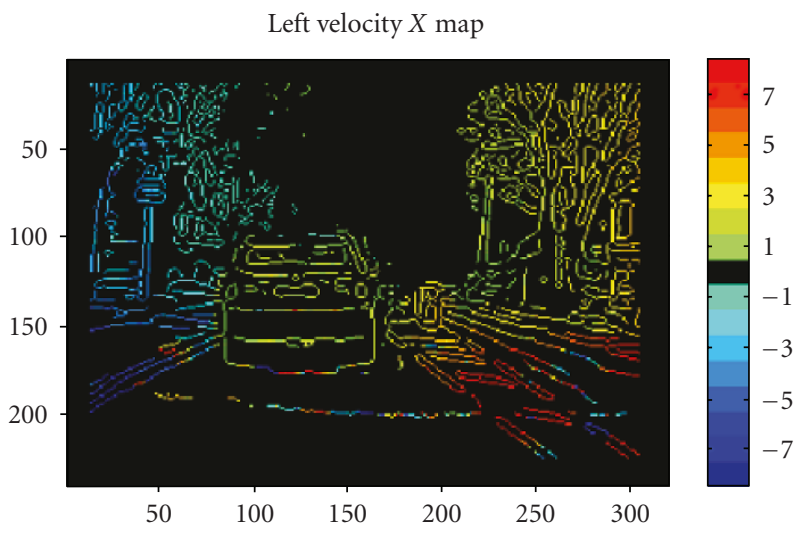

(a) Horizontal motion displacement of left images

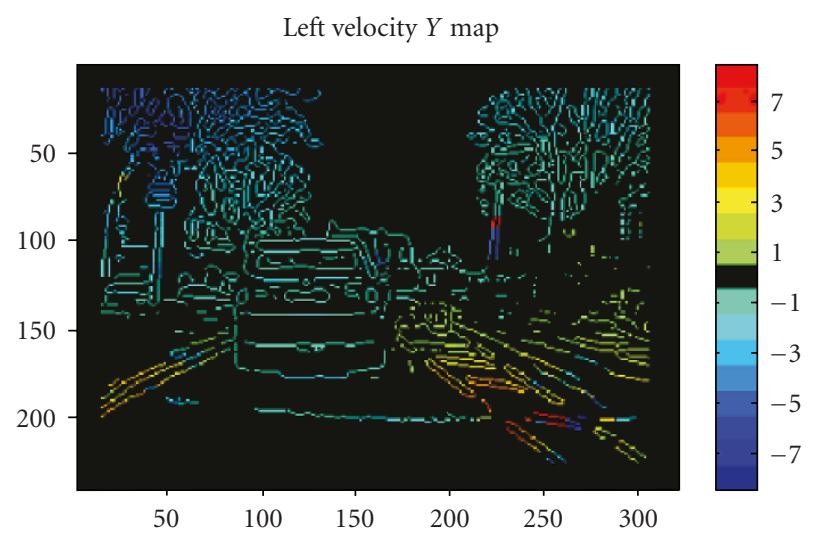

(c) Vertical motion displacement of right images

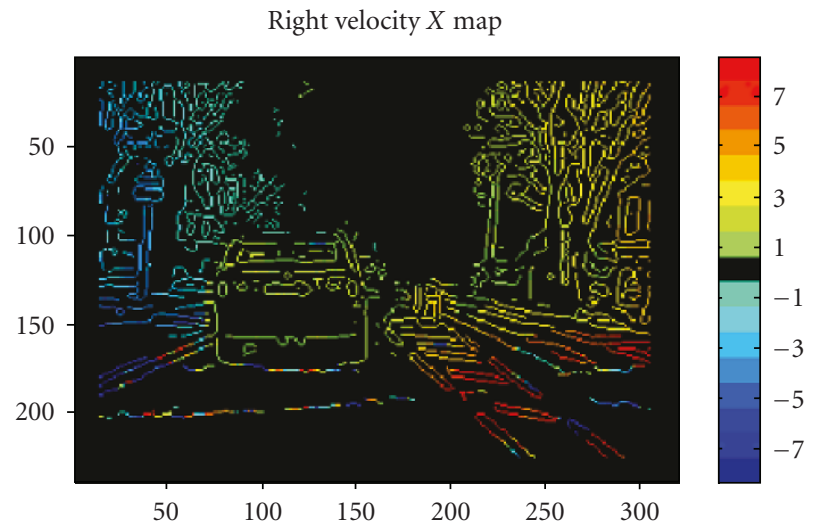

(b) Horizontal motion displacement of right images

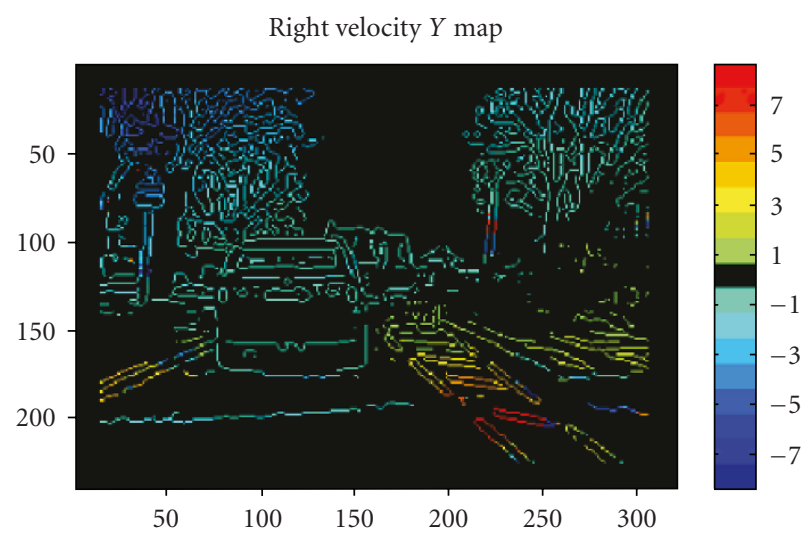

(d) Vertical motion displacement of right images

FIgURE 3: Optic flows for left and right images using edge-indexed crosscorrelation matching.

search range, was set to 8 in both directions. For a zero displacement, the colour is set to black. Actually, the calculated displacements have subpixel accuracy. Comparing Figure 3(a) to 3(b) and 3(c) to 3(d), we can find the motion displacements of the left and right image sequences have very similar distributions even if some noisy matching has occurred, which implies that the optic flows can be used as extra constraints for stereo matching. 


\subsection{Using optic flows as extra constraints for stereo matching}

Our previous work only used the normalized cross correlation coefficient as a measure to assess the correlation of the point pair. The point pair with the maximum correlation coefficient is considered as the correspondence points. In fact, this is not always true because of an intensity difference between the two images caused by lighting angle, iris or exposure gain of the cameras. The disparity map generated by this method for the image pair at frame 60 is shown in Figure 4(a). It can be seen that some colour discontinuity occurs in the same object indicating mismatching points.

By introducing optic flows as extra constraints, we are defining multiple measures for stereo matching. As well as the normalized cross correlation coefficient, two other measures are defined as the differences of the horizontal and vertical displacements of two points to be assessed. Actually, texture measure is also very important to assess the matching quality in some nontextured regions of the image [1]. In this study, we only conduct stereo matching for edge points where the image patches are normally textured. Furthermore, as we only want to assess the effect of optic flows on the stereo matching, we do not consider the texture measure.

Applying the epipolar line constraint, we can search the correspondence point in the same row. For a seed point at position $\left(i_{l}, j\right)$ in the left edge image and a candidate point at position $\left(i_{r}, j\right)$ in the right image, the differences of the motion displacements in horizontal and vertical directions, $\operatorname{MVD}_{x}\left(i_{l}, j\right)$ and $\operatorname{MVD}_{y}\left(i_{l}, j\right)$, are defined as

$$
\begin{aligned}
& \operatorname{MVD}_{x}\left(i_{l}, j\right)=\left|\operatorname{MV}_{x}\left(i_{l}, j\right)-\operatorname{MV}_{x}\left(i_{r}, j\right)\right|, \\
& \operatorname{MVD}_{y}\left(i_{l}, j\right)=\left|\operatorname{MV}_{y}\left(i_{l}, j\right)-\operatorname{MV}_{y}\left(i_{r}, j\right)\right|,
\end{aligned}
$$

where $\mathrm{MV}_{x}$ and $M V_{y}$ are the horizontal and vertical motion displacements obtained in Section 2.1.

Accordingly, we define a three-dimensional measure vector $M\left(i_{l}, j\right)$ as follows:

$$
M\left(i_{l}, j\right)=\left[\operatorname{MVD}_{x}\left(i_{l}, j\right) \operatorname{MVD}_{y}\left(i_{l}, j\right) 1-\operatorname{coef}\left(i_{l}, j\right)\right]^{T},
$$

where $\operatorname{coef}(i, j)$ is the normalized cross correlation coefficient for the seed point at position $\left(i_{l}, j\right)$ in the left image. To achieve the best matching, $\operatorname{MVD}_{x}(i, j)$ and $\operatorname{MVD}_{y}(i, j)$ should be minimised while $\operatorname{coef}(i, j)$ should be maximised.

The matching error $E\left(i_{l}, i_{r}, j\right)$ between pixel $\left(i_{l}, j\right)$ in the left image and pixel $\left(i_{r}, j\right)$ in the right image is defined as a weighted sum of the individual measures

$$
E\left(i_{l}, i_{r}, j\right)=\sum_{k} w_{k}\left[M\left(i_{l}, j\right)\right]_{k}
$$

where $k$ represents the measure vector index. The selection of the weighting is objective and empirical. The weighting for the third measure $(1-\operatorname{coef}(i, j))$ should have a greater value than the motion displacement differences. For an edge point $\left(x_{l}, y\right)$ in the left image, the correspondence point in the

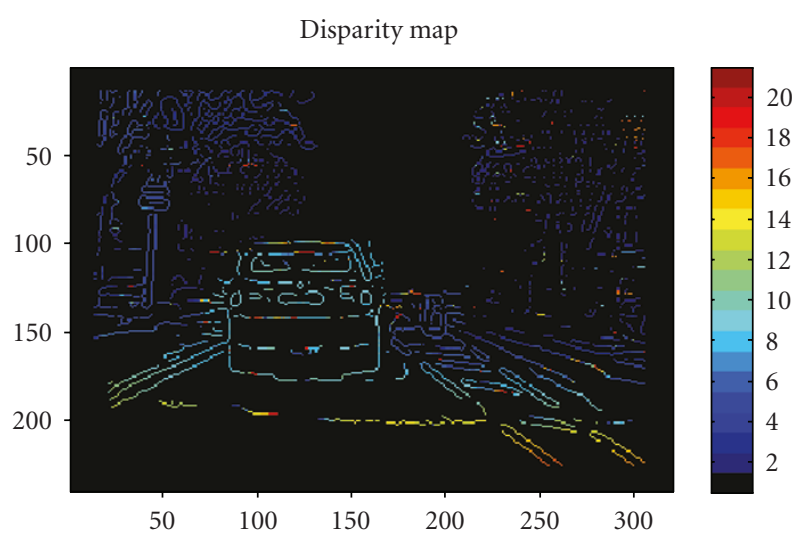

(a) No fusion

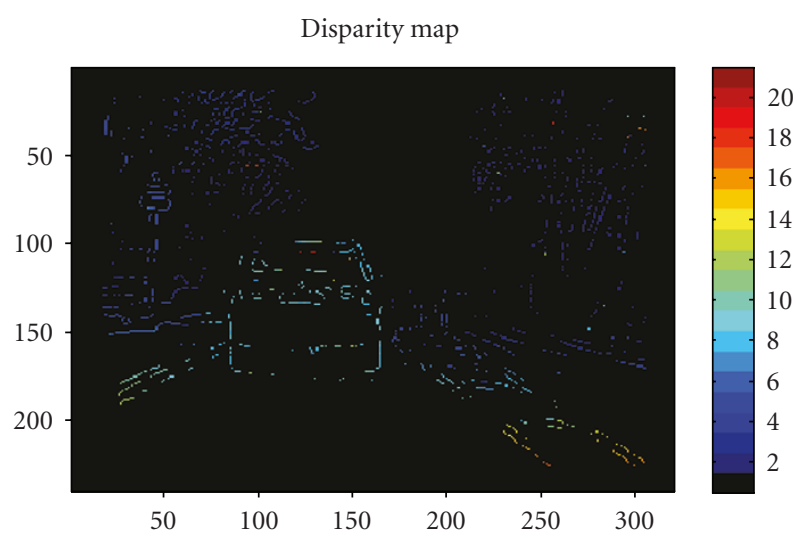

(b) With fusion

FIGURE 4: Disparity maps with fusion and no fusion with optic flows.

right image is the one $\left(x m_{r}, y m\right)$ generating the minimum matching error, and $x_{l}-x m_{r}$ gives out the disparity. The disparity map generated by this method for the image pair at frame 60 is shown in Figure 4(b). The comparison between Figures 4(a) and 4(b) indicates that the disparity map obtained from the fusion method is much cleaner with many false matching points eliminated.

\section{FUSION USING A KALMAN FILTER FOR 3D OBJECT TRACKING}

\subsection{Modelling}

For a point $P(X Y Z)$ in the world coordinate, the state vector to be tracked is defined by $\mathrm{SV}=$

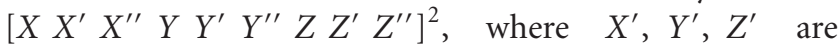
the first-order derivative of $X, Y, Z$ that is $3 \mathrm{D}$ speeds of the point, $X^{\prime \prime}, Y^{\prime \prime}, Z^{\prime \prime}$ are the second-order derivative of $X, Y, Z$, that is $3 \mathrm{D}$ accelerations of the point. Assuming the point to be tracked follows a purely translational movement, the prediction equation of the Kalman filter can be defined 
as the object motion equation

$$
\mathrm{SV}_{k+1}=A \cdot \mathrm{SV}_{k}+\xi_{k}
$$

where $k$ is the sampling index, $\xi_{k}$ process noise,

$$
A=\left[\begin{array}{ccccccccc}
1 & T & 0.5 T^{2} & 0 & 0 & 0 & 0 & 0 & 0 \\
0 & 1 & T & 0 & 0 & 0 & 0 & 0 & 0 \\
0 & 0 & 1 & 0 & 0 & 0 & 0 & 0 & 0 \\
0 & 0 & 0 & 1 & T & 0.5 T^{2} & 0 & 0 & 0 \\
0 & 0 & 0 & 0 & 1 & T & 0 & 0 & 0 \\
0 & 0 & 0 & 0 & 0 & 1 & 0 & 0 & 0 \\
0 & 0 & 0 & 0 & 0 & 0 & 1 & T & 0.5 T^{2} \\
0 & 0 & 0 & 0 & 0 & 0 & 0 & 1 & T \\
0 & 0 & 0 & 0 & 0 & 0 & 0 & 0 & 1
\end{array}\right]
$$

and $T$ the sampling interval. The Kalman filter also needs a measurement vector and measurement equations to update the prediction.

For an ideal pinhole camera model, the projection point $p(x y)$ of the point $P(X Y Z)$ in the image plane can be expressed as

$$
\left[\begin{array}{l}
x \\
y
\end{array}\right]=\left[\begin{array}{l}
m \cdot \frac{X}{Z} \\
m \cdot \frac{Y}{Z}
\end{array}\right],
$$

where $m$ is the camera constant regarding the focal length and the pixel size. This equation indicates the relationship between real 3D world coordinates and 2D image coordinates. It can be seen from (6) that three variables $x, y$, and $Z$ are crucial for updating prediction equation (4). Therefore, we can define the measurement vector as MV = $\left[\begin{array}{lll}x & y & Z\end{array}\right]^{T}$. It is known that the distance $Z$ can be measured from the stereovision technique. Horizontal and vertical coordinates $(x, y)$ can be measured because horizontal and vertical displacements $\Delta x$ and $\Delta y$ between the imagescan be measured by the motion analysis, and the initial position $\left(x_{c}, y_{c}\right)$ of the tracking point are known from (17). Thus, the Kalman filter provides a natural way to fuse stereovision with motion analysis for three-dimensional object tracking.

The measurement equation can be written as

$$
\mathrm{MV}_{k}=C \cdot \mathrm{SV}_{k}+\eta_{k}
$$

where $\eta_{k}$ is the measurement noise,

$$
C=\left[\begin{array}{ccccccccc}
\frac{m}{Z} & 0 & 0 & 0 & 0 & 0 & 0 & 0 & 0 \\
0 & 0 & 0 & \frac{m}{Z} & 0 & 0 & 0 & 0 & 0 \\
0 & 0 & 0 & 0 & 0 & 0 & 1 & 0 & 0
\end{array}\right]
$$

Equation (4) forms a complete object motion equation. The process estimation noise only comes from $X^{\prime \prime}, Y^{\prime \prime}, Z^{\prime \prime}$, that is $3 \mathrm{D}$ accelerations. Therefore, the process noise $\xi_{k}$ in (4) can be modelled as $\xi_{k}=\left[\begin{array}{lllllllll}0 & 0 & a_{x} & 0 & 0 & a_{y} & 0 & 0 & a_{z}\end{array}\right]^{T} . a_{x}, a_{y}$, and $a_{z}$ are considered as Gaussian white noise with a mean value of zero. Their variances are $\partial_{a x}^{2}, \partial_{a y}^{2}$, and $\partial_{a z}^{2}$. The processing noise covariance $Q_{k}$ is

$$
\begin{aligned}
Q_{k} & =E\left[\xi_{k} \cdot \xi_{k}^{T}\right] \\
& =\left[\begin{array}{ccccccccc}
0 & 0 & 0 & 0 & 0 & 0 & 0 & 0 & 0 \\
0 & 0 & 0 & 0 & 0 & 0 & 0 & 0 & 0 \\
0 & 0 & \partial_{a x}^{2} & 0 & 0 & 0 & 0 & 0 & 0 \\
0 & 0 & 0 & 0 & 0 & 0 & 0 & 0 & 0 \\
0 & 0 & 0 & 0 & 0 & 0 & 0 & 0 & 0 \\
0 & 0 & 0 & 0 & 0 & \partial_{a y}^{2} & 0 & 0 & 0 \\
0 & 0 & 0 & 0 & 0 & 0 & 0 & 0 & 0 \\
0 & 0 & 0 & 0 & 0 & 0 & 0 & 0 & 0 \\
0 & 0 & 0 & 0 & 0 & 0 & 0 & 0 & \partial_{a z}^{2}
\end{array}\right] .
\end{aligned}
$$

The measurement noise $\eta_{k}$ consists of $\eta x_{k}, \eta y_{k}$, and $\eta Z_{k}$. Their variance $\partial_{x}^{2}, \partial_{y}^{2}, \partial_{Z}^{2}$ can be calculated from measurement data. Measurement noise covariance $R_{k}$ are

$$
\begin{aligned}
R_{k} & =E\left[\eta_{k} \cdot \eta_{k}^{T}\right] \\
& =\left[\begin{array}{ccc}
\partial_{x}^{2} & 0 & 0 \\
0 & \partial_{y}^{2} & 0 \\
0 & 0 & \partial_{Z}^{2}
\end{array}\right] .
\end{aligned}
$$

The state vector SV contains three groups of state variables $\left(X X^{\prime} X^{\prime \prime}\right),\left(Y Y^{\prime} Y^{\prime \prime}\right)$, and $\left(Z Z^{\prime} Z^{\prime \prime}\right)$, which are not directly related to each other. Thus, we decouple the state vector SV into three groups; each group with two state variables. Correspondingly, (4) and (7) are also split into three groups. Each group forms a linear Kalman filter with the computation reduced from $6^{3}$ to $3^{*} 2^{3}$. For example, for the third group, the longitudinal distance and speed, the state vector is $\mathrm{SV}=\left[\begin{array}{lll}Z & Z^{\prime} & Z^{\prime \prime}\end{array}\right]^{T}$, and the measurement vector is $\mathrm{MV}=[Z]$. The prediction and measurement equations keep the same format as (4) and (7) with some changes in matrix $A$ and $C$, that is

$$
\begin{aligned}
A_{3} & =\left[\begin{array}{ccc}
1 & T & 0.5 T^{2} \\
0 & 1 & T \\
0 & 0 & 1
\end{array}\right], \\
C_{3} & =\left[\begin{array}{lll}
1 & 0 & 0
\end{array}\right] .
\end{aligned}
$$

Kalman filtering is an iteration process, and can be described with five equations consisting of two time update and three measurement update equations. The time update equations for the filter are

$$
\begin{aligned}
\mathrm{SV}_{k+1}^{-} & =A \cdot \mathrm{SV}_{k}, \\
P_{k+1}^{-} & =A \cdot P_{k} \cdot A^{T}+Q_{k},
\end{aligned}
$$

where $P_{k}$ is the variance of the estimation error. The three measurement update equations are

$$
\begin{aligned}
K_{k+1} & =\frac{P_{k+1}^{-} \cdot C^{T}}{\left[C \cdot P_{k+1}^{-} \cdot C^{T}+R_{k}\right]}, \\
\mathrm{SV}_{k+1} & =\mathrm{SV}_{k+1}^{-}+K_{k+1}\left[\mathrm{MV}_{k+1}-C \cdot \mathrm{SV}_{k+1}^{-}\right], \\
P_{k+1} & =\left[1-K_{k+1} \cdot C\right] P_{k+1}^{-},
\end{aligned}
$$




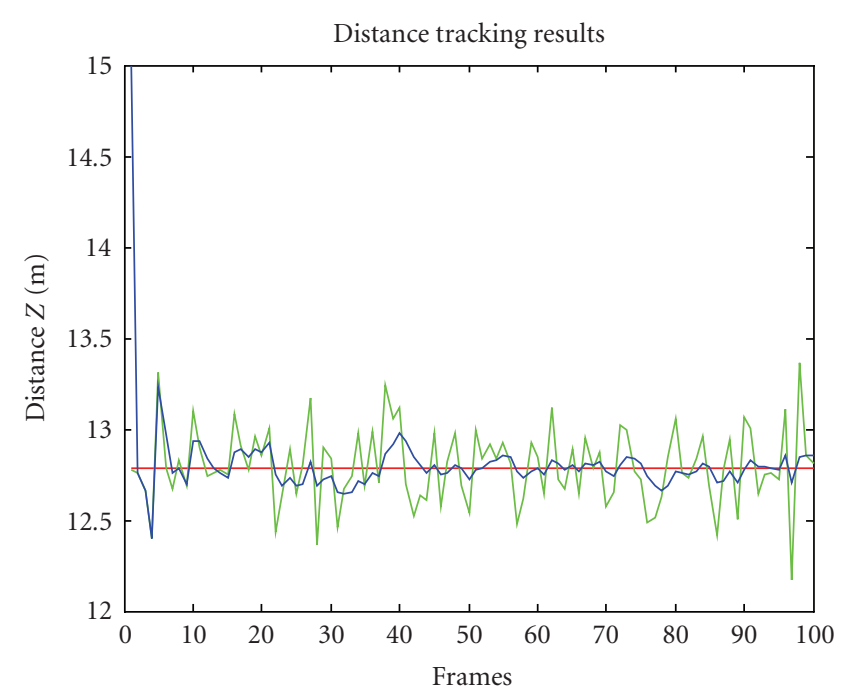

(a) The longitudinal distance

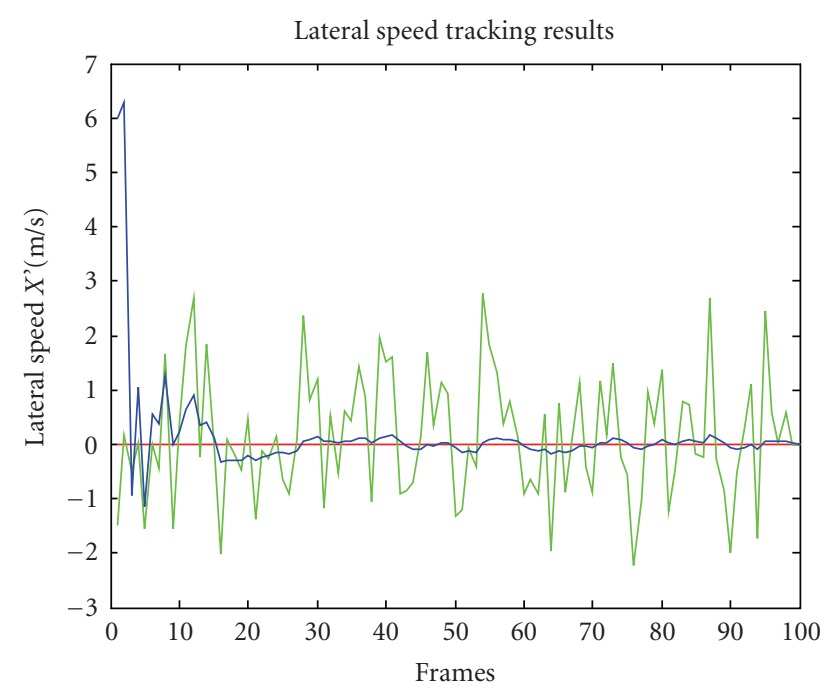

(c) The lateral speed

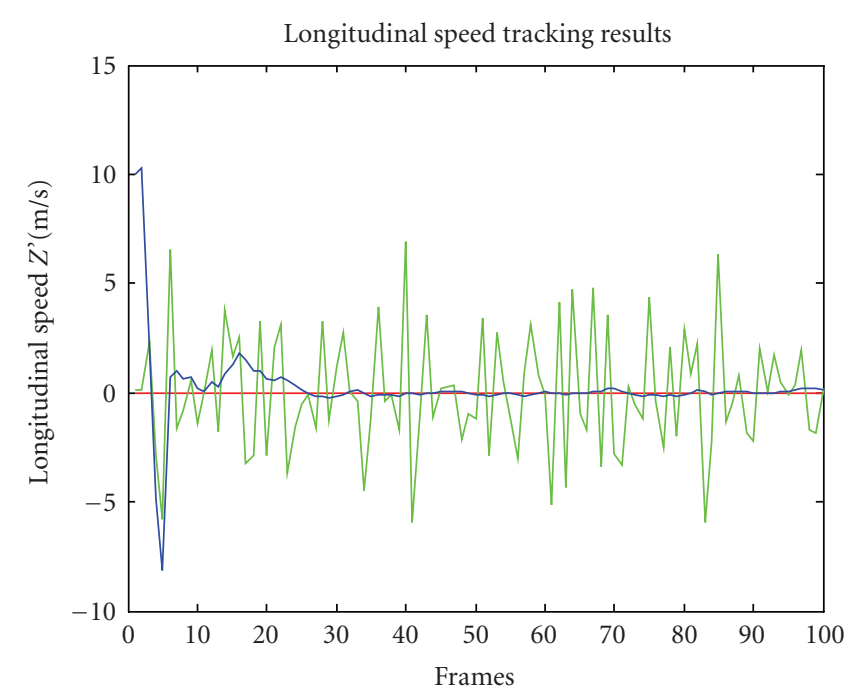

(b) The longitudinal speed

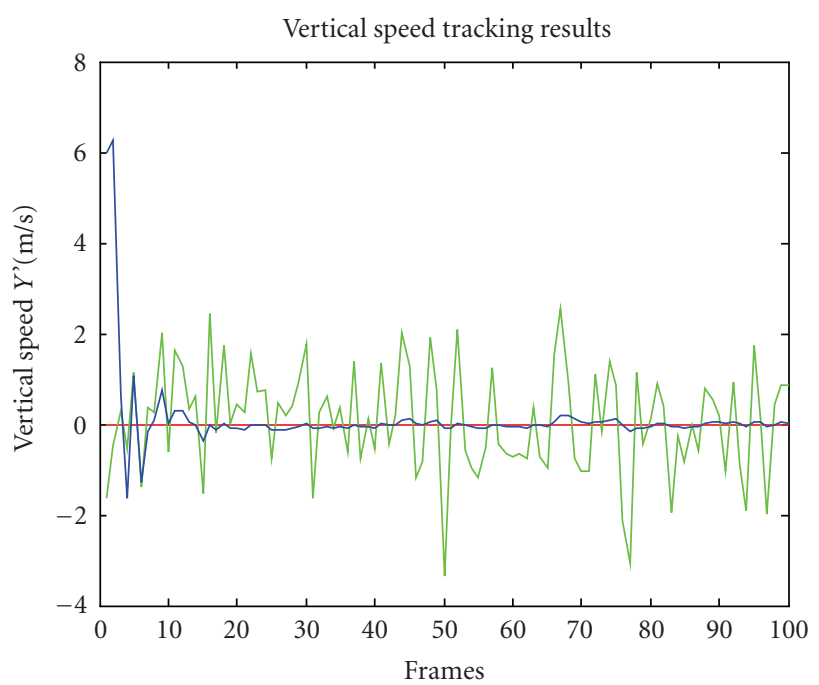

(d) The vertical speed

FIGURE 5: Experiment 1: tracking results by using a Kalman filter as a mixer (red: the true state of the system, green: the measurement results, blue: the estimation of the Kalman filter).

where $K$ is the Kalman gain, $R_{k}=\partial_{Z}^{2}$, and

$$
Q_{k}=\left[\begin{array}{ccc}
0 & 0 & 0 \\
0 & 0 & 0 \\
0 & 0 & \partial_{a z}^{2}
\end{array}\right] .
$$

$\mathrm{SV}_{k+1}$ gives the tracking results for the longitudinal distance and speed.

The other two groups of state variables, the lateral and vertical movements, have the same iteration equations with a small change in matrix $C$, that is, $C_{1}=C_{2}=\left[\begin{array}{lll}m / Z & 0 & 0\end{array}\right]$, which requires knowing the longitudinal distance $Z$. Therefore, in practice, we first calculate the third group of the variables and then use the obtained results for the other two groups of variables.

\subsection{Experiments}

Object longitudinal distance $(Z)$ is detected by the stereovision method explained in $[1,2]$. In this method, an object to be detected is segmented as a point cluster in the depth map. The point cluster is then remapped back into the original image, which generates an object image region $R_{\text {obj }}$. The centroid point of an object detected is selected as the tracking point. The centroid point $\left(x_{c}, y_{c}\right)$ is defined as

$$
\begin{aligned}
& x_{c}=\frac{\sum_{i \in R_{\mathrm{obj}}} \sum_{j \in R_{\mathrm{obj}}} \cdot G(i, j)}{\sum_{i \in R_{\mathrm{obj}}} \sum_{j \in R_{\mathrm{obj}}} G(i, j)}, \\
& y_{c}=\frac{\sum_{i \in R_{\mathrm{obj}}} \sum_{j \in R_{\mathrm{obj}}} j \cdot G(i, j)}{\sum_{i \in R_{\mathrm{obj}}} \sum_{j \in R_{\mathrm{obj}}} G(i, j)},
\end{aligned}
$$




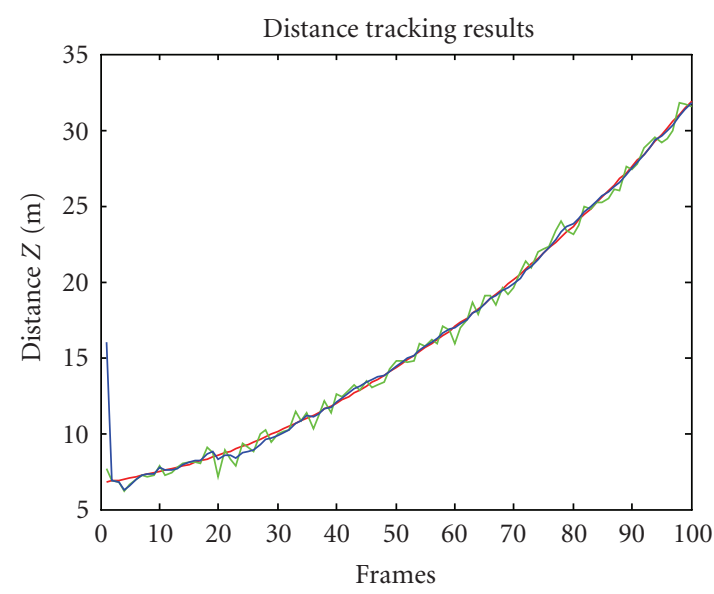

(a) The longitudinal distance

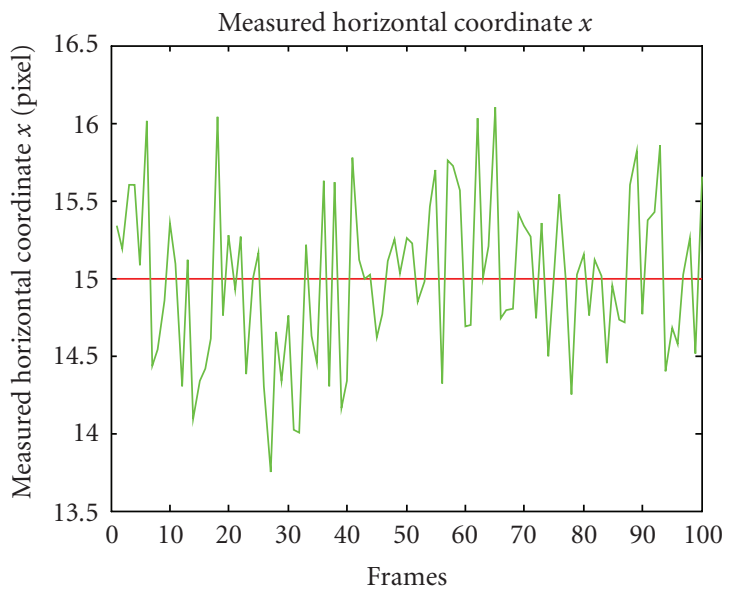

(c) Measured horizontal coordinate $x$

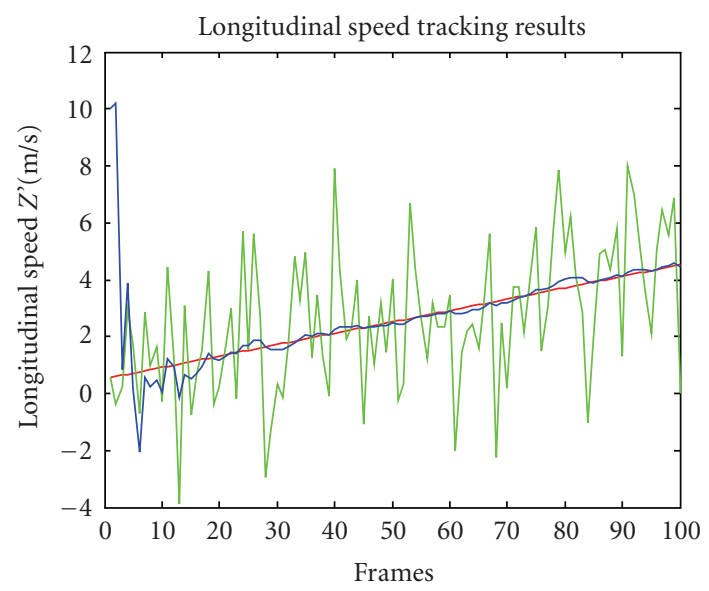

(b) The longitudinal speed

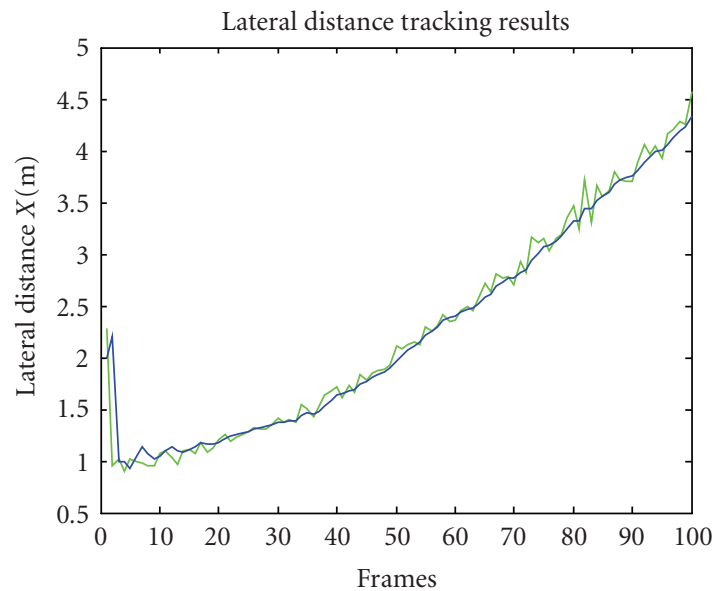

(d) The lateral distance $X$

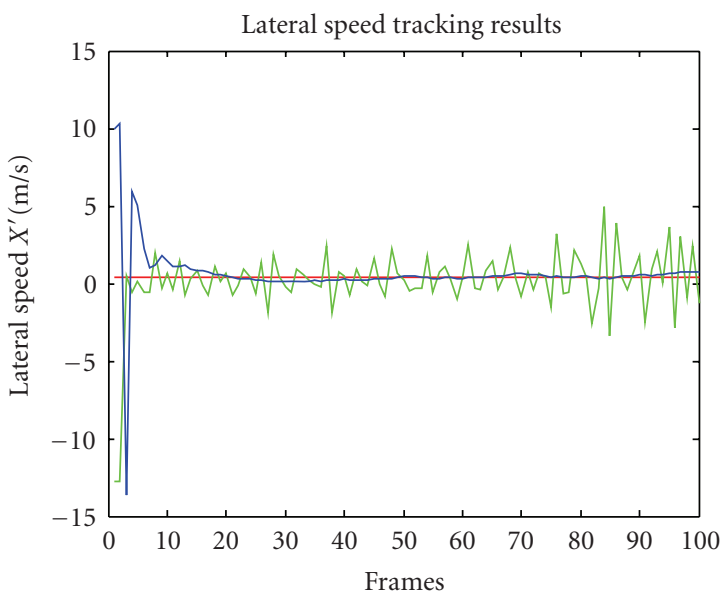

(e) The lateral speed $X^{\prime}$

FIGURE 6: Experiment 2: tracking results by using Kalman filter as a mixer (red: the true state of the system, green: the measurement results, blue: the estimation of the Kalman filter).

where

$$
G(i, j)= \begin{cases}1 & \text { if } i \in R_{\mathrm{obj}} \cap j \in R_{\mathrm{obj}} \\ 0 & \text { other. }\end{cases}
$$

Actually, we only need to locate the centroid point the first time the object has been detected. Consequently, this point will have been used to calculate the optic flows of the object.

Two experiments have been conducted to verify the algorithms. Each experiment captures a pair of image 
sequences containing 100 frames as shown in Figure 1. When using (12)-(15) to calculate the optimal estimation, two parameters, the measurement noise covarianceand the processing noise covariance must be prespecified. In this work, the measurement covariance noise was evaluated from the measurement data $\partial_{x}^{2}=\partial_{y}^{2}=0.04$ and $\partial_{Z}^{2}=$ 0.05 . The process noise was modelled as a Gaussian white noise with a mean value of zero and a standard deviation of 0.03 , therefore, processing noise covariance $\partial_{a x}^{2}=\partial_{a y}^{2}=\partial_{a z}^{2} \approx 0.001$. Initial values of the state vector $\mathrm{SV}$ and the variance of the estimation error $P_{k}$ required by the iteration process were randomly set. Our experiments demonstrate that the tracking results are not very sensitive to the settings of the process noise or the initial values.

Experiment 1 gives the simplest scenario where the car under detection is moving $12.8 \mathrm{~m}$ ahead of the equipped car at a relative speed of zero in both longitudinal and lateral directions. Thus, the longitudinal and lateral distances between the two cars are kept constant. The tracking results of the longitudinal distance and relative speed are shown in Figures 5(a) and 5(b). The lateral and vertical relative speeds are shown in Figures 5(c) and 5(d). In the figures, the red line is the true state of the system, the green curve measurement results, and the blue curve the estimation of the Kalman filter. It can be seen that after about 10 frames, the estimation converges on the true values. Furthermore, we find that the tracking results are more stable and closer to the true values than the measurement results.

Experiment 2 covers a more generic case where the car under detection is moving away from the equipped car with a constant acceleration of $0.4 \mathrm{~m} / \mathrm{s}^{2}$ and an initial speed of $0.556 \mathrm{~m} / \mathrm{s}$ in the longitudinal direction. In the lateral direction, the car under detection is moving from the equipped car at a speed of $0.4 \mathrm{~m} / \mathrm{s}$. Figures $6(\mathrm{a})$ and 6 (b) shows the tracking results of the longitudinal distance and speed. The longitudinal distance between the two cars varies from $6.8 \mathrm{~m}$ to $32.1 \mathrm{~m}$ in a form of a parabolic curve. The longitudinal speed increases linearly from $0.556 \mathrm{~m} / \mathrm{s}$ to $4.556 \mathrm{~m} / \mathrm{s}$. Figure 6(c) shows the measured horizontal coordinate $x$. Correspondingly, the lateral distance $X$ between the two cars and the tracking results are shown in Figure 6(d). It can be seen that the horizontal coordinate varies around a mean value of 15 pixel while the lateral distance varies in a form of an approximate parabolic curve, affected by the measured longitudinal distance. Lateral speed tracking results are displayed in Figure 6(e). As in the previous experiment, all estimation values converge on the true values after about 10 frames. Furthermore, we also find that the tracking results are more stable and closer to the true values than the measurement results, which indicates the designed Kalman filter is able to generate an optimal estimation. The results of experiment 2 demonstrate that the designed Kalman filter is able to dynamically track the distance and speed in both longitudinal and lateral directions while the car under detection is accelerating away.

\section{CONCLUSIONS}

In this paper, we proposed two algorithms for fusing stereovision and motion analysis. The first algorithm is to fuse the optic flows into stereo matching, where the optic flows are used as extra constraints for stereo matching. The matching error is defined as the weighted sum of multiple measures including the normalized cross correlation coefficient, and differences of horizontal and vertical motion displacements. The experimental results demonstrated that the disparity map obtained from the fusion method is much cleaner than the normal method with many false matching points eliminated. The second fusion algorithm is to use a Kalman filter as a mixer to combine the measurements generated from stereovision and motion analysis for 3-dimensional object tracking. A decoupling strategy has been designed to achieve a simplified linear Kalman filter, which greatly reduces the computation complexity and burden. The experimental results have demonstrated that the tracking results converge on the true values. Furthermore, the tracking results are more stable and closer to the true values than the raw measurement results, indicating that the proposed Kalman filter is able to generate optimal estimations for $3 \mathrm{D}$ object motion parameters.

\section{REFERENCES}

[1] Y. Huang, S. Fu, and C. Thompson, "Stereovision-based object segmentation for automotive applications," EURASIP Journal on Applied Signal Processing, vol. 2005, no. 14, pp. 2322-2329, 2005.

[2] Y. Huang, "Obstacle detection in urban traffic using stereovision," in Proceedings of the 8th IEEE International Conference on Intelligent Transportation Systems (ITSC '05), pp. 357-362, Vienna, Austria, September 2005.

[3] U. Franke and A. Joos, "Real-time stereo vision for urban traffic scene understanding," in Proceedings of IEEE Intelligent Vehicles Symposium (IV '00), pp. 273-278, Dearborn, Mich, USA, October 2000.

[4] Y. Kimura, T. Kato, and M. Ohta, "Stereo vision for obstacle detection," in Proceedings of the 13th World Congress \& Exhibition on Intelligent Transportation Systems and Services, London, UK, October 2006.

[5] T. Suzuki and T. Kanada, "Measurement of vehicle motion and orientation using optical flow," in Proceedings of the IEEE/IEEJ/JSAI International Conference on Intelligent Transportation Systems (ITSC '99), pp. 25-30, Tokyo, Japan, October 1999.

[6] Z. Hu and K. Uchimura, "Tracking cycle: a new concept for simultaneous tracking of multiple moving objects in a typical traffic scene," in Proceedings of IEEE Intelligent Vehicles Symposium (IV '00), pp. 233-239, Dearborn, Mich, USA, October 2000.

[7] N. Ancona, "A fast obstacle detection method based on optical flow," in Proceedings of the 2nd European Conference on Computer Vision (ECCV'92), pp. 267-271, Santa Margherita Ligure, Italy, May 1992.

[8] D. Willersinn and W. Enkelmann, "Robust obstacle detection and tracking by motion analysis," in Proceedings of IEEE International Conference on Intelligent Transportation Systems (ITSC '97), pp. 717-721, Boston, Mass, USA, November 1997. 
[9] A. M. Waxman and J. H. Duncan, "Binocular image flows: steps towards stereo-motion fusion," IEEE Transactions on Pattern Analysis and Machine Intelligence, vol. 8, no. 6, pp. 715729, 1986.

[10] L. Li and J. H. Duncan, "3-D translational motion and structure from binocular image flows," IEEE Transactions on Pattern Analysis and Machine Intelligence, vol. 15, no. 7, pp. 657-667, 1993.

[11] W. Wang and J. H. Duncan, "Recovering the threedimensional motion and structure of multiple moving objects from binocular image flows," Computer Vision and Image Understanding, vol. 63, no. 3, pp. 430-446, 1996.

[12] Y. Zhang and C. Kambhamettu, "On 3-D scene flow and structure recovery from multiview image sequences," IEEE Transactions on Systems, Man, and Cybernetics B, vol. 33, no. 4, pp. 592-600, 2003.

[13] G. Sudhir, S. Banarjee, K. Biswas, and R. Bahl, "Cooperative integration of stereopsis and optical flow computation," Journal of the Optical Society of America A, vol. 12, no. 12, pp. 2564-2572, 1995.

[14] S. P. Clifford and N. M. Nasrabadi, "Integration of stereo vision and optical flow using Markov randomfields," in Proceedings of IEEE International Conference on Neural Networks, vol. 1, pp. 577-584, San Diego, Calif, USA, July 1988.

[15] A. P. Tirumalai, B. G. Schunck, and R. C. Jain, "Dynamic stereo with self-calibration," IEEE Transactions on Pattern Analysis and Machine Intelligence, vol. 14, no. 12, pp. 1184-1189, 1992.

[16] T. Dang, C. Hoffmann, and C. Stiller, "Fusing optical flow and stereo disparity for object tracking," in Proceedings of the 5th IEEE International Conference on Intelligent Transportation Systems (ITSC '02), pp. 112-117, Singapore, September 2002.

[17] B. McCane, K. Novins, D. Crannitch, and B. Galvin, "On benchmarking optical flow," Computer Vision and Image Understanding, vol. 84, no. 1, pp. 126-143, 2001. 